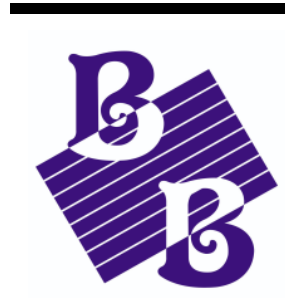

BioBacta

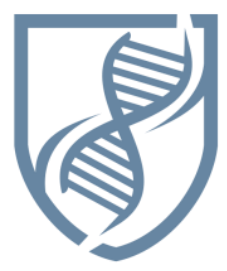

Journal of Bioscience and Applied Research

www.jbaar.org

\title{
Evaluation of the cardioprotective effect of l-carnitine and silymarin in cancer patients receiving anthracycline-containing chemotherapy
}

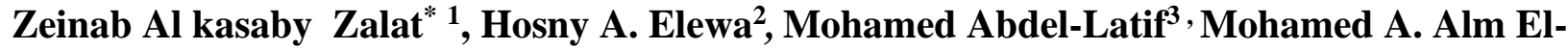 Din $^{4}$, Neeven A. Kohaf ${ }^{5}$}

1. Assoc. Professor and Head of Clinical Pharmacy Department, Faculty of Pharmacy, Al-Azhar University, Cairo, Egypt. (Pharm.D., Ph.D.) (zeinabalkasaby.pharmg@azhar.edu.eg)

2. Assoc. professor and Head of Pharmacy Practice Department, Faculty of Pharmacy, Horus University, Dominate City, Egypt. (Pharm.D, Ph.D.) helewa@ horus.edu.eg

3. Professor and Head of Clinical Pharmacy Department, Faculty of Pharmacy, Assiut University, Assiut, Egypt. (Ph.D.)

4. Assoc. Professor, Department of Clinical Oncology, Faculty of Medicine, Tanta University, Tanta, Egypt. (MD)

5. Master degree in pharmaceutical sciences, Faculty of Pharmacy, Al-Azhar University, Cairo, Egypt. (M.Sc.)

*Corresponding author: Zeinab Al kasaby Zalat (zeinabalkasaby.pharmg@azhar.edu.eg)

Running title: Cardioprotective effect of l-carnitine and silymarin

Received: July 20, 2020. Accepted: September 30, 2020. Published: October 18, 2020

DOI: 10.21608/jbaar.2020.119755

\begin{abstract}
Aim: Anthracycline-induced cardiotoxicity is the most common constraint of its use in the treatment of various types of cancer. This study aimed to investigate the benefits of the addition of the 1-carnitine / silymarin to anthracycline chemotherapy in patients with breast cancer. Methods: 83 patients were recruited from Clinical Oncology Department, Tanta University, Egypt, then prospectively randomized to receive their anthracyclinecontaining therapeutic regimen, control group $(n=33)$, or anthracycline plus 1-carnitine, 1-carnitine group $(n=25)$, or anthracycline plus silymarin, silymarin group $(n=25)$. Blood samples were collected at the beginning and after 6 months to measure LDH, CK-MB, cTn I, Anticardiolipin IgG, Fe, ferritin, and TIBC and $\%$ of saturation. $\% \mathrm{EF}$ was documented. Data were statistically analyzed by ANOVA and paired t-test. $P<0.05$ was statistically significant. Results: The addition of l-carnitine to anthracycline chemotherapy has a significantly improved EF\% ( $\mathrm{P}=0.003)$, Anticardiolipin $\operatorname{IgG}(P=0.001)$, ferritin $(\mathrm{P}=0.001)$, and TIBC $(\mathrm{P}=0.011)$. The supplementation with silymarin to anthracycline chemotherapy had a statistically significant decrease in Anticardiolipin IgG $(\mathrm{P}=0.000)$, iron $(\mathrm{P}=0.001)$, ferritin $(\mathrm{P}=0.001)$, TIBC $(\mathrm{P}=0.007)$, and \% saturation $(\mathrm{P}=0.001)$. Silymarin group showed a significant decrease in iron profile compared to the l-carnitine group. Conclusion: The co-administration of l-carnitine or silymarin with anthracycline chemotherapy represents a new therapeutic strategy for better control of anthracycline-induced cardiotoxicity. Silymarin
\end{abstract}


resulted in more beneficial effects on the iron profile compared to l-carnitine with anthracycline or anthracycline chemotherapy alone.

Keywords: Anthracycline chemotherapy; cardiotoxicity; 1-carnitine; silymarin

\section{Introduction}

Over the past decades, progresses in cancer diagnosis and treatment have improved the life expectancy of countless patients worldwide. Anthracycline is one of the most worldwide used anticancer drugs, the discovery of doxorubicin was near to 1960s as powerful anticancer anthracycline antibiotics, it's one of the essential chemotherapy that used to treat cancer. [1]

Anthracyclines are useful anticancer drugs because it's used with other chemotherapeutic drugs in the treatment of breast cancer, acute leukemia, Hodgkin's and non-Hodgkin's lymphomas, bone and soft tissue sarcoma, Wilms cancer, and many other malignant neoplasms. [2]

In particular, anticancer drugs are toxic to the cardiovascular system. The mechanisms of anthracycline cardiotoxicity are multi-factorial. These drugs "induce multiple forms of cellular injuries by free radical production, alter nucleic acid biology by intercalation into DNA, and modulate intracellular signaling that leads to cell death and disruption of homeostatic process, such as sarcomere maintenance"[3]. There is growing evidence that cardiotoxicity from anticancer drugs may occur at any time after completing chemotherapy. Therefore, early detection and prevention of anthracyclines induced cardiotoxicity are particularly important and have already aroused more attention. [4]

Measurement of cardio-specific biomarkers can be a valid diagnostic tool for early identification, assessment, and monitoring of cardiotoxicity. The advantages of biomarkers include being minimally invasive, less expensive than echocardiography or nuclear techniques, and can easily be repeated without irradiation of the patient. Moreover, the interpretation of the results does not depend on the expertise of the operator, thus avoiding the possibility of interobserver variability [5]

The prevention of anthracycline-induced cardiotoxicity is an important challenge in cancer survivorship. In trying to prevent or attenuate the side effects of doxorubicin administration, several strategies have been followed as dosage optimization, synthesis, and use of analogs or combined therapy like antioxidants. the combination of the drug delivery together with an antioxidant in order to reduce the toxic effects of doxorubicin by decreasing the oxidative stress without interference with its antitumor properties [6] Dexrazoxane has been used in cancer patients to prevent anthracycline-related cardiotoxicity since the 1980s. The use of dexrazoxane as a cardioprotectant has been associated with increased risks of infection, myelosuppression, and second primary malignancies (SPMs), therefore the CHMP published a recommendation for the restrictions on dexrazoxane use in both children and adults with cancer [7]. So, substances, such as silymarin and l-carnitine can be protective for cardiomyocytes because of their antioxidant effects and cell membrane stabilizing as well as radical scavenging potency (3).

L-carnitine is a cofactor required for the transformation of free long-chain fatty acids into acylcarnitines, and for their subsequent transport into 
the mitochondrial matrix, where they undergo betaoxidation for cellular energy production. Conditions that appear to benefit from exogenous supplementation of L-carnitine include anorexia, chronic fatigue, cardiovascular disease, diphtheria, hypoglycemia, male infertility, muscular myopathies, and Rett syndrome [8]. Long-term supplementation of L-carnitine ( $2 \mathrm{~g}$ daily) for the treatment of heart failure caused by dilated cardiomyopathy resulted in improvement in survival rate, ejection fraction, Weber classification, maximal time of cardiopulmonary exercise test, peak VO2 consumption, arterial and pulmonary blood pressure, and cardiac output. [9]

Silymarin is a non-toxic natural polyphenolic flavonoid extracted from seeds of the plant milk thistle (Silybum marianum) which is an ancient medicinal plant for the treatment of various liver diseases [10]. Due to its strong antioxidant and tissue regenerative properties; 'Silymarin 'is being studied as a hepatic, neural, renal, and cardiac protective ingredient. [11]. Silymarin could be beneficial in oncology patients, especially for the treatment of the side effects of anticancer chemotherapy [8]. Silymarin has also an anti-cancer effect in common cancers, such as lung, prostatic, colon, breast, bladder, as well as, hepatocellular carcinoma. [12]

Therefore; the objective of the present work is to evaluate the protective effects of l-carnitine or silymarin as cardioprotective agents against the side effects of anthracycline chemotherapy. Moreover, the study aimed to assess the effects of both treatment modalities on biochemical markers LDH, CK-MB, Troponin I, Anticardiolipin IgG, Iron, ferritin, and TIBC and calculated parameters $\% \mathrm{EF}$ and \% of saturation.

Material, patients, and methods Study patients
The study participants were recruited from the Oncology Department, Tanta University Hospital, Egypt. The study was designed and conducted in compliance with the ethical principles of Good Clinical Practice Guidelines and the Declaration of Helsinki [13]. The study protocol was approved by the National Research Ethics Committee [Tanta University Ethical Committee for Clinical Research] approval code (32551/09/2018). Informed written consent was obtained from all individual participants included in the study.

Patients accepted in this study fulfilled the following criteria: cancer patients receiving anthracycline chemotherapy in their protocol alone (without any cardioprotective agent), aged 20-60, and both male and female patients were included.

Patients were excluded from the study: patients with a history of heart failure, arrhythmia, history of cardiac catheterizations or, history of angina, uncontrolled hypertension and uncontrolled diabetes, patients with impaired liver function tests, previous anthracycline-containing regimens and any cardiotoxic chemotherapy regimens, previous history of chest wall irradiation. Brain metastasis, pregnant patients, and patients who refused informed consent, patients who could not be sampled serially, or had bypass surgery, which precluded sampling throughout their hospital stay were also excluded.

\section{Materials}

Drugs:

L-carnitine ${ }^{\circledR} \quad 500 \quad \mathrm{mg}$ capsules obtained from (MEPACO)

Silymarin (Legalon ® $140 \mathrm{mg}$ capsule obtained from (MEDA).

Kits: LDH kits Manufactured by BioSystem. S.A Company, Spain, CK-MB kits Manufactured by BioSystem. S.A Company, Spain, IRONCHROMAZUROL kits Manufactured by BioSystem. 
S.A Company, Spain, Enzyme-Linked Immunosorbent Assay (ELISA) kits (Manufactured by ORGENTEC Diagnostika GmbH Company, Germany used to assay anti-cardiolipin IgG. EnzymeLinked Immunosorbent Assay (ELISA) kits Manufactured by Accu-Bind Company, the USA used to assay Troponin-I (cTnI). Ferritin EnzymeLinked Immunosorbent Assay (ELISA) kits Manufactured by Accu-Bind Company, USA was used for ferritin assay Total iron-binding capacity (TIBC) kits Manufactured by BioSystem. S.A Company, Spain was used to assay TIBC.

\section{Study design}

This study was a parallel randomized (simple randomization; odd number took silymarin and even number took 1 carnitine) controlled prospective openlabel one. Out of 105 patients with different types of cancer screened for eligibility, 8 patients did not complete their treatment course (shifted to another protocol), 8 refused to be enrolled in the study and 6 showed bad drug adherence, and randomized to control group ( $n=41)$, L-carnitine group $(n=32)$ and, Silymarin group $(n=32)$, finally, eighty-three eligible patients were recruited 33,25 , and 25 patients in the control group, L-carnitine group, and, Silymarin group respectively, completed the study as shown in Figure 1. L-carnitine group received anthracyclinecontaining chemotherapy in a dose of $50 \mathrm{mg} / \mathrm{m} 2$ plus L-carnitine 3 gm L-carnitine ${ }^{\circledR}$ capsules obtained from (MEPACO) was taken PO one day before the chemotherapeutic cycle and $1 \mathrm{gm}$ /day during the following 21 days. Silymarin group received anthracycline-containing chemotherapy in a dose of $50 \mathrm{mg} / \mathrm{m} 2$ plus Silymarin as cardioprotective agent.140 mg (Legalon (® $140 \mathrm{mg}$ capsule obtained from (MEDA). Silymarin was taken PO once daily after meals during the chemotherapeutic cycle. The treatment period was 6 months.
All patients were interviewed for a complete history and clinical examination, which were carried out by a qualified physician from Clinical Oncology Department, Tanta University Hospital. All patients were counseled to continue the same medications that they were receiving before the enrollment in this study. All subjects were assessed monthly throughout the treatment period to assess adherence to the study protocol and to report any dropout or adverse events.

\section{Biochemical analysis}

Samples collection

Venous blood samples $(5 \mathrm{ml})$ were obtained from all the patients before and after the treatment course ( 6 months). The serum supernatant was separated immediately from the blood by centrifugation for 15 minutes at $3000 \mathrm{rpm}$. The serum was divided into five parts coded and stored at $-80{ }^{\circ} \mathrm{C}$ for biochemical analysis.

\section{Assay of LDH, CK-MB, and Iron}

LDH kits Manufactured by BioSystem. S.A Company, Spain used to assay LDH spectrophotometrically.CK-MB was assayed using CK-MB kits Manufactured by BioSystem. S.A Company, Spain. Iron-Chromazurol kits Manufactured by BioSystem. S.A Company, Spain was used to assay iron.

\section{Assay of anti-cardiolipin IgG, Troponin I, TIBC, and ferritin}

Enzyme-Linked Immunosorbent Assay (ELISA) kits (Manufactured by ORGENTEC Diagnostika GmbH Company, Germany used to assay anti-cardiolipin IgG. Enzyme-Linked Immunosorbent Assay (ELISA) kits Manufactured by Accu-Bind Company, USA used to assay Troponin-I (cTnI). Ferritin Enzyme-Linked Immunosorbent Assay (ELISA) kits Manufactured by Accu-Bind Company, USA was used for ferritin assay total iron-binding capacity (TIBC) kits Manufactured by BioSystem. S.A Company, Spain was used to assay TIBC. 


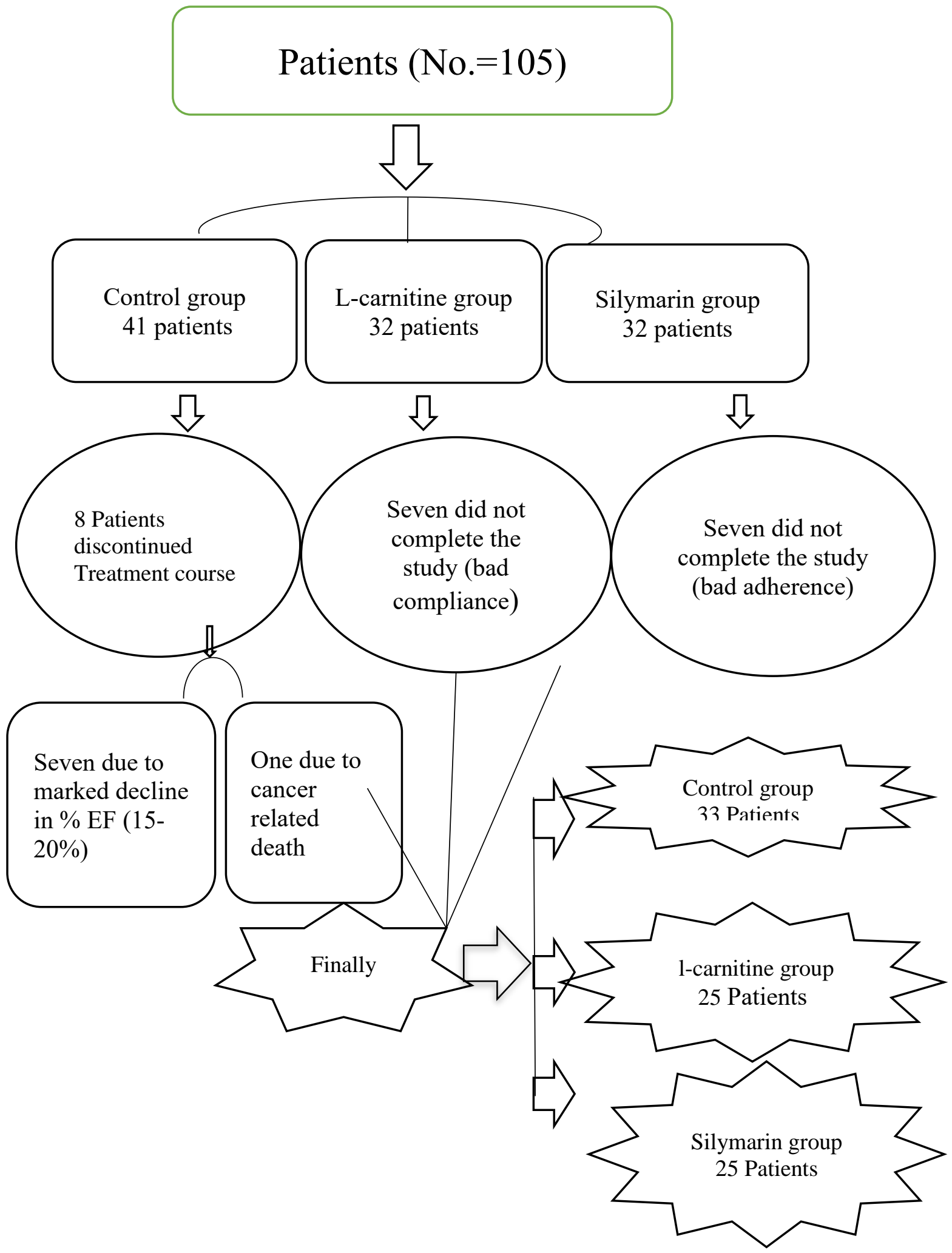

Figure 1: Patients' enrollment flow chart 


\section{Statistical analysis}

Data were tabulated and analyzed using SPSS statistical package version 26.0, IBM Corporation Software Group, USA, and Microsoft office professional plus (excel) 2019. Data are expressed as the mean value ( \pm standard deviation). One-way analysis of variance test (one-way ANOVA) followed by LSD post hoc test was used to assess any significant difference between the three groups. Paired t-test was used to assess any significant difference within each group at baseline and after chemotherapy. All probability values presented were two-tailed and $p \leq 0.05$ was considered statistically significant.

\section{Results}

\section{Demographic data in the studied groups}

The age ranged from 20-60 in all groups. There were two males in each group and 31, 23, and 23 female patients in the control group, 1-carnitine group, and silymarin group respectively Table 1 . There were 29 , 21 , and 21 breast cancer patients. There were 2, 1 , and, 2 patients with Hodgkin lymphoma (HL), and 2, 3 , and 2 non-Hodgkin lymphomas (NHL) patients in control, 1-carnitine and silymarin groups respectively. There were 17, 18, and 13 breast adjuvant patients, and 2, 2, and 6 breast neoadjuvant patients in control, L-carnitine, and Silymarin groups respectively. There were 2,1 and 2 breast metastatic patients in control, 1carnitine and silymarin groups respectively Table 2 .

Table 3 showed the different therapeutic regimens used. The dose of Adriamycin was $50 \mathrm{mg} / \mathrm{m}^{2}$ in all treatment protocols. There was a non- significant difference in all demographic data between the studied groups $\mathrm{P} \geq 0.05$. There were no significant differences between studied groups in baseline clinical data as shown in Table 4.

Comparison between studied groups at baseline and after chemotherapy
Table 5 shows that when comparing $\% \mathrm{EF}$ at baseline and after anthracycline chemotherapy (Figure 2) there is a significant decrease within the control group, a significant increase within l-carnitine group $\mathrm{P}=0.00$, $\mathrm{P}=0.002$ respectively and a non-significant difference within silymarin group $\mathrm{P}=0.817$. when comparing LDH (U/L) at baseline and after anthracycline chemotherapy, there is a significant increase within the control group $\mathrm{P}=0.002$ while within the l-carnitine group there is a significant decrease $\mathrm{P}=0.003$ and in the silymarin group, there are a-significant decrease $\mathrm{P}=0.006$, when comparing CK-MB and troponin I (Figure 3) at baseline and after anthracycline chemotherapy, there is a significant increase within the control group $\mathrm{P}=0.003$ and a significant decrease within treated groups. Figure 4 shows ACL IgG (U/L) at baseline and after anthracycline chemotherapy, there is a significant increase within the control group $\mathrm{P}=0.000$ while within the l-carnitine group and silymarin there is a significant decrease within groups $\mathrm{P}=0.002$ and 0.0001 respectively, as shown in Table 5

Comparison between studied groups at baseline and after 6 months of chemotherapy regarding iron profile

Table 6 shows that when comparing iron $(\mu \mathrm{g} / \mathrm{dl})$ at baseline and after anthracycline chemotherapy, there is a significant difference within the control group and silymarin $\mathrm{P}=0.003$ and $\mathrm{P}=0.001$ respectively while within the 1-carnitine group there is a non-significant difference within group $\mathrm{P}=0.928$, when comparing Ferritin $(\mathrm{ng} / \mathrm{ml})$ at baseline and after anthracycline chemotherapy, there was a significant difference within the control group, l-carnitine and silymarin $\mathrm{P}=0.003, \mathrm{P}=0.003$ and $\mathrm{P}=0.001$ respectively. when comparing TIBC $(\mu \mathrm{g} / \mathrm{dl})$ at baseline and after anthracycline chemotherapy, there is a significant difference within the control group, 1-carnitine and silymarin group $\mathrm{P}=0.000, \mathrm{P}=0.011$, and $\mathrm{P}=0.007$ respectively, when comparing $\%$ of saturation at 
baseline and after anthracycline chemotherapy, there carnitine group and a significant increase within are a significant decrease within silymarin group $\mathrm{P}=$ control group $\mathrm{P}=0.002$.

0.001 and non-significant differences within the 1-

Table 1: Demographic data of the studied groups at baseline

\begin{tabular}{|c|c|c|c|c|c|c|c|}
\hline \multirow[b]{2}{*}{ Variable } & \multicolumn{6}{|c|}{ Groups } & \multirow[b]{2}{*}{ P-value } \\
\hline & \multicolumn{2}{|c|}{$\begin{array}{l}\text { Control Group } \\
\mathrm{N}=33\end{array}$} & \multicolumn{2}{|c|}{$\begin{array}{l}\text { l-carnitine group } \\
\mathrm{N}=25\end{array}$} & \multicolumn{2}{|c|}{$\begin{array}{l}\text { Silymarin group } \\
\mathrm{N}=25\end{array}$} & \\
\hline Age (years) Mean \pm SD & \multicolumn{2}{|c|}{$44.455 \pm 9.47$} & \multicolumn{2}{|c|}{$45.64 \pm 9.941$} & \multicolumn{2}{|c|}{$44.68 \pm 12.44$} & 0.61 \\
\hline Sex: & $\mathrm{N}$ & $\%$ & $\mathrm{~N}$ & $\%$ & $\mathrm{~N}$ & $\%$ & \\
\hline $\begin{array}{l}\text { Male } \\
\text { Female }\end{array}$ & 31 & $\begin{array}{l}(6.06 \%) \\
(93.93 \%)\end{array}$ & $\begin{array}{l}2 \\
23\end{array}$ & $\begin{array}{l}(8 \%) \\
(92 \%)\end{array}$ & $\begin{array}{l}2 \\
23\end{array}$ & $\begin{array}{l}(8 \%) \\
(92 \%)\end{array}$ & 0.727 \\
\hline
\end{tabular}

Age represented in mean \pm slandered deviation

Data represented in number and percent. $\mathrm{p} \leq 0.05$ value considered significant

Table 2: Distribution of patients regarding pathology and stage in the studied groups

\begin{tabular}{|c|c|c|c|c|c|c|c|}
\hline \multirow{3}{*}{$\begin{array}{l}\text { Variable } \\
\text { Pathologv: }\end{array}$} & \multicolumn{6}{|c|}{ Groups } & \multirow{3}{*}{$P$-Value } \\
\hline & \multicolumn{2}{|c|}{ Control } & \multicolumn{2}{|c|}{ L- Carnitine } & \multicolumn{2}{|c|}{ Silymarin } & \\
\hline & $\mathrm{N}$ & $\%$ & $\mathrm{~N}$ & $\%$ & $\mathrm{~N}$ & $\%$ & \\
\hline Breast cancer & 29 & $(87.87 \%)$ & 21 & $(84 \%)$ & 21 & $(84 \%)$ & \\
\hline NHL & 4 & $(12.12 \%)$ & 4 & $(16 \%)$ & 4 & $(16 \%)$ & 0.942 \\
\hline \multicolumn{8}{|l|}{ Stage: } \\
\hline Breast adjuvant & 17 & $(51.51 \%)$ & 18 & $(72 \%)$ & 13 & $(52 \%)$ & \\
\hline Breast neoadjuvant & 2 & $(6.06 \%)$ & 2 & $(8 \%)$ & 6 & $(24 \%)$ & \\
\hline Breast metastatic & 2 & $(6.06 \%)$ & 1 & $(4 \%)$ & 2 & $(8 \%)$ & \\
\hline NHL II & 2 & $(6.06 \%)$ & 4 & $(16 \%)$ & 1 & $(4 \%)$ & 0742 \\
\hline NHL III & 2 & $(6.06 \%)$ & 0 & 0 & 3 & $(12 \%)$ & \\
\hline
\end{tabular}

NHL: Non-Hodgkin lymphoma. Data represented in number and percent. $\mathrm{p} \leq 0.05$ value considered significant Age represented in mean \pm slandered deviation

Data represented in number and percent. $\mathrm{p} \leq 0.05$ value considered significant 
Table 3: Distribution of patients according to therapeutic regimen

\begin{tabular}{|c|c|c|c|c|c|c|c|}
\hline \multirow{3}{*}{ Variable } & \multicolumn{6}{|c|}{ Groups } & \multirow{3}{*}{ P-Value } \\
\hline & \multicolumn{2}{|c|}{ Control } & \multicolumn{2}{|c|}{ L-Carnitine } & \multicolumn{2}{|c|}{ Silymarin } & \\
\hline & $\mathbf{N}$ & $\%$ & $\mathbf{N}$ & $\%$ & $\mathbf{N}$ & $\%$ & \\
\hline \multicolumn{8}{|c|}{ Chemotherapy regimen: } \\
\hline $\mathbf{A C}$ & 9 & $(27.2 \%)$ & 12 & $(48 \%)$ & 12 & $(48 \%)$ & \\
\hline FAC & 20 & $(60 \%)$ & 9 & $(36 \%)$ & 9 & $(36 \%)$ & 0.406 \\
\hline CHOP & 4 & $(12.12 \%)$ & 4 & $(16 \%)$ & 4 & $(16 \%)$ & \\
\hline
\end{tabular}

Data represented in number and percent. $\mathrm{p} \leq 0.05$ value considered significant.$A C$ : Adriamycin + cyclophosphamide

FAC: 5 -flourouracil +Adriamycin +cyclophosphamide. CHOP: Cyclophosphamide +Adriamycin +Oncovin

+ Prednisone. The significance level was set at $\mathrm{p} \leq 0.05$.

Table 4: Baseline data of the studied groups

\begin{tabular}{|c|c|c|c|c|}
\hline \multirow[b]{2}{*}{ Variable } & \multicolumn{3}{|c|}{ Groups } & \multirow[b]{2}{*}{ P-value } \\
\hline & $\begin{array}{c}\text { Control group } \\
\qquad \mathbf{N}=\mathbf{3 3}\end{array}$ & $\begin{array}{c}\text { l-carnitine group } \\
\mathrm{N}=25\end{array}$ & $\begin{array}{c}\text { Silymarin group } \\
\qquad \mathrm{N}=\mathbf{2 5}\end{array}$ & \\
\hline$\% \mathrm{EF}$ & $67.6 \pm 0.036$ & $66.7 \pm 0.045$ & $66.7 \pm 0.0438$ & 0.637 \\
\hline LDH (U/I) & $367.5 \pm 83.97$ & $361 \pm 142.7$ & $363.48 \pm 140.2$ & 0.979 \\
\hline CK-MB (IU/I) & $15.32 \pm 4.51$ & $15.08 \pm 4.88$ & $15.144 \pm 2.89$ & 0.973 \\
\hline Troponin I (ng/ml) & $0.145 \pm 0.054$ & $0.16 \pm 0.058$ & $0.145 \pm 0.0505$ & 0.560 \\
\hline ACL IGg (CU) & $1.396 \pm 0.52$ & $1.444 \pm 0.54$ & $1.772 \pm 0.65$ & 0.924 \\
\hline Iron $\mu \mathrm{g} / \mathrm{dl}$ & $89.06 \pm 15.14$ & $88.9 \pm 14.15$ & $88.7 \pm 16.2$ & 0.997 \\
\hline Ferritin ng/ml & $202.125 \pm 78.82$ & $200.7 \pm 77.5$ & $202.8 \pm 78.69$ & 0.984 \\
\hline TIBC $\mu \mathrm{g} / \mathrm{dl}$ & $296 \pm 72.12$ & $298 \pm 71.6$ & $287 \pm 72.3$ & 0.987 \\
\hline Saturation \% & $32.38 \pm 11.1$ & $31.8 \pm 11.2$ & $32.9 \pm 10.8$ & 0.995 \\
\hline
\end{tabular}

Data are presented as mean ( \pm standard deviation). The significance level was set at $p \leq 0.05$. EF: Ejection fraction, LDH: Lactate dehydrogenase, CK-MB: Creatine kinase and ACL IGg: anti-cardiolipin IGg, TIBC: total iron binding capacity 
Table 5: Comparison between studied groups at base line and after 6 months of chemotherapy

\begin{tabular}{|c|c|c|c|c|c|c|}
\hline \multirow{3}{*}{ Variable } & \multicolumn{6}{|c|}{ Groups } \\
\hline & \multicolumn{2}{|c|}{$\begin{array}{l}\text { Control Group } \\
\mathrm{N}=33\end{array}$} & \multicolumn{2}{|c|}{$\begin{array}{l}\text { l-carnitine Group } \\
\mathrm{N}=25\end{array}$} & \multicolumn{2}{|c|}{$\begin{array}{l}\text { Silymarin Group } \\
\mathrm{N}=25\end{array}$} \\
\hline & At baseline & After 6 months & At baseline & After 6 months & At baseline & After 6 months \\
\hline$\% \mathrm{EF}$ & $67.6 \pm 0.036$ & $63.18 \pm 0.044$ & $66.7 \pm 0.045$ & $68.56 \pm 0.031$ & $66.7 \pm 0.0438$ & $66.8 \pm 0.0367$ \\
\hline P-value & \multicolumn{2}{|c|}{$0.001^{*}$} & \multicolumn{2}{|c|}{$0.003^{*}$} & \multicolumn{2}{|c|}{0.817} \\
\hline LDH (U/L) & $367.5 \pm 83.97$ & $592.2 \pm 214.5$ & $361 \pm 142.7$ & $214 \pm 82.8$ & $363.48 \pm 140.2$ & $259.72 \pm 103.47$ \\
\hline P-value & \multicolumn{2}{|c|}{$0.001 *$} & \multicolumn{2}{|c|}{$0.002^{*}$} & \multicolumn{2}{|c|}{$0.006^{*}$} \\
\hline $\begin{array}{l}\text { CK-MB } \\
(\mathrm{IU} / \mathrm{L})\end{array}$ & $15.32 \pm 4.51$ & $33.27 \pm 10.33$ & $15.08 \pm 4.88$ & $10.37 \pm 4.093$ & $15.144 \pm 2.89$ & $7.936 \pm 3.0309$ \\
\hline $\mathrm{P}$-value & \multicolumn{2}{|l|}{$0.001^{*}$} & \multicolumn{2}{|c|}{$0.002 *$} & \multicolumn{2}{|c|}{$0.001 *$} \\
\hline $\begin{array}{l}\text { Troponin I } \\
(\mathrm{ng} / \mathrm{ml})\end{array}$ & $0.145 \pm 0.054$ & $0.46 \pm 0.162$ & $0.16 \pm 0.058$ & $0.094 \pm 0.025$ & $0.145 \pm 0.0505$ & $0.0894 \pm 0.0338$ \\
\hline $\mathrm{P}$-value & \multicolumn{2}{|c|}{$0.003 *$} & \multicolumn{2}{|l|}{$0.002 *$} & \multicolumn{2}{|c|}{$0.001 *$} \\
\hline $\begin{array}{l}\text { ACL IgG } \\
(\mathrm{CU})\end{array}$ & $1.396 \pm 0.52$ & $10.63 \pm 4.04$ & $1.444 \pm 0.54$ & $2.021 \pm 0.76$ & $1.772 \pm 0.65$ & $1.11 \pm 0.4107$ \\
\hline P-value & \multicolumn{2}{|c|}{$0.002 *$} & \multicolumn{2}{|l|}{$0.001^{*}$} & \multicolumn{2}{|l|}{$0.003^{*}$} \\
\hline
\end{tabular}

Data are presented as mean ( \pm standard deviation). The significance level was set at $\mathrm{p}<0.05$. $*$ : statistically significant difference: Ejection fraction, LDH: Lactate dehydrogenase, CK-MB: Creatine kinase and ACL IGg: anti-cardiolipin Igg 
Table 6: Comparison between studied groups at base line and after 6 months of chemotherapy regarding iron profile

\begin{tabular}{|c|c|c|c|c|c|c|}
\hline & \multicolumn{2}{|c|}{$\begin{array}{l}\text { Control Group } \\
\mathrm{N}=33\end{array}$} & \multicolumn{2}{|c|}{$\begin{array}{l}\text { l-carnitine Group } \\
\mathrm{N}=25\end{array}$} & \multicolumn{2}{|c|}{$\begin{array}{l}\text { Silymarin Group } \\
\mathrm{N}=25\end{array}$} \\
\hline & At baseline & After 6 months & At baseline & After 6 months & At baseline & After 6 months \\
\hline Iron $(\mu \mathrm{g} / \mathrm{dl})$ & $89.06 \pm 15.14$ & $102.4 \pm 17.5$ & $88.9 \pm 14.15$ & $91.52 \pm 23.95$ & $88.7 \pm 16.2$ & $73.36 \pm 14.36$ \\
\hline$P$-value & \multicolumn{2}{|l|}{$0.003^{*}$} & \multicolumn{2}{|l|}{0.920} & \multicolumn{2}{|c|}{$0.001^{*}$} \\
\hline $\begin{array}{l}\text { Ferritin(ng/ } \\
\text { ml) }\end{array}$ & $202.12 \pm 78.82$ & $256.8 \pm 93.25$ & $200.7 \pm 77.5$ & $95.56 \pm 19.27$ & $202.8 \pm 78.69$ & $68.52 \pm 27$ \\
\hline$P$-value & \multicolumn{2}{|c|}{$0.003^{*}$} & \multicolumn{2}{|l|}{$0.000^{*}$} & \multicolumn{2}{|c|}{$0.000^{*}$} \\
\hline $\begin{array}{l}\text { TIBC } \\
(\mu \mathrm{g} / \mathrm{dl})\end{array}$ & $296 \pm 72.12$ & $238.62 \pm 55.17$ & $298 \pm 71.6$ & $365.1 \pm 49.2$ & $287 \pm 72.3$ & $374.8 \pm 35.1$ \\
\hline$P$-value & \multicolumn{2}{|l|}{$0.000 *$} & \multicolumn{2}{|l|}{$0.011^{*}$} & \multicolumn{2}{|c|}{$0.007 *$} \\
\hline $\begin{array}{l}\% \text { of } \\
\text { saturation }\end{array}$ & $32.83 \pm 11.1$ & $44.8 \pm 11.64$ & $31.8 \pm 11.2$ & $25.24 \pm 6.4$ & $32.9 \pm 10.8$ & $19.7 \pm 3.97$ \\
\hline$P$-value & \multicolumn{2}{|l|}{$0.000 *$} & \multicolumn{2}{|l|}{0.052} & \multicolumn{2}{|c|}{$0.001 *$} \\
\hline
\end{tabular}

Data are presented as mean ( \pm standard deviation). The significance level was set at $\mathrm{p} \leq 0.05 . *$ : statistically significant difference.

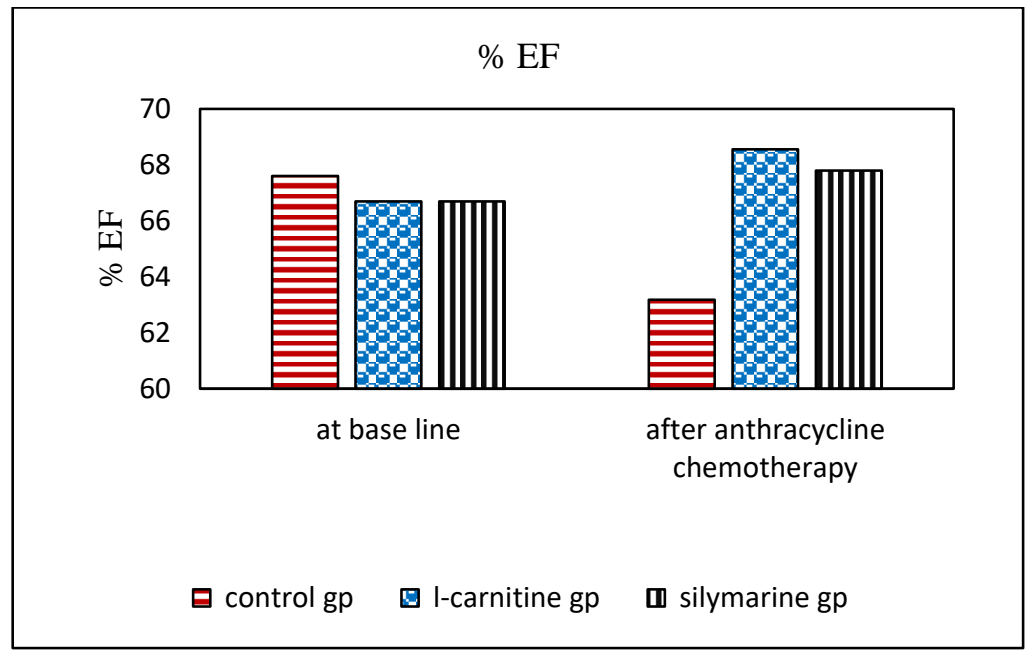

Figure 2: Comparison between studied groups regarding \% EF at base line and after 6 months of chemotherapy 


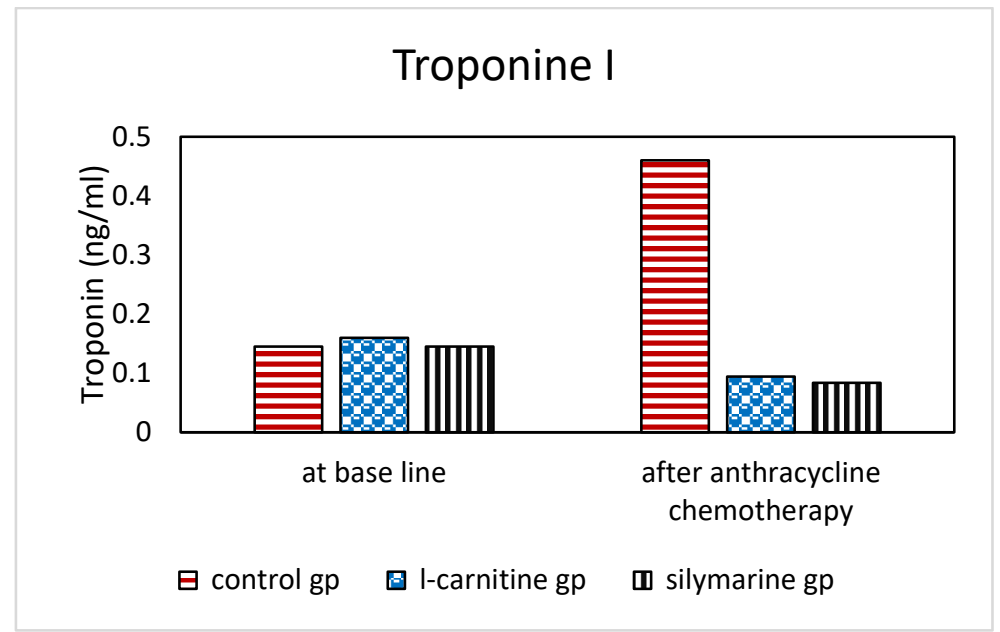

Figure 3: Comparison between studied groups regarding troponin I (ng/ml) at base line and after 6 months of chemotherapy

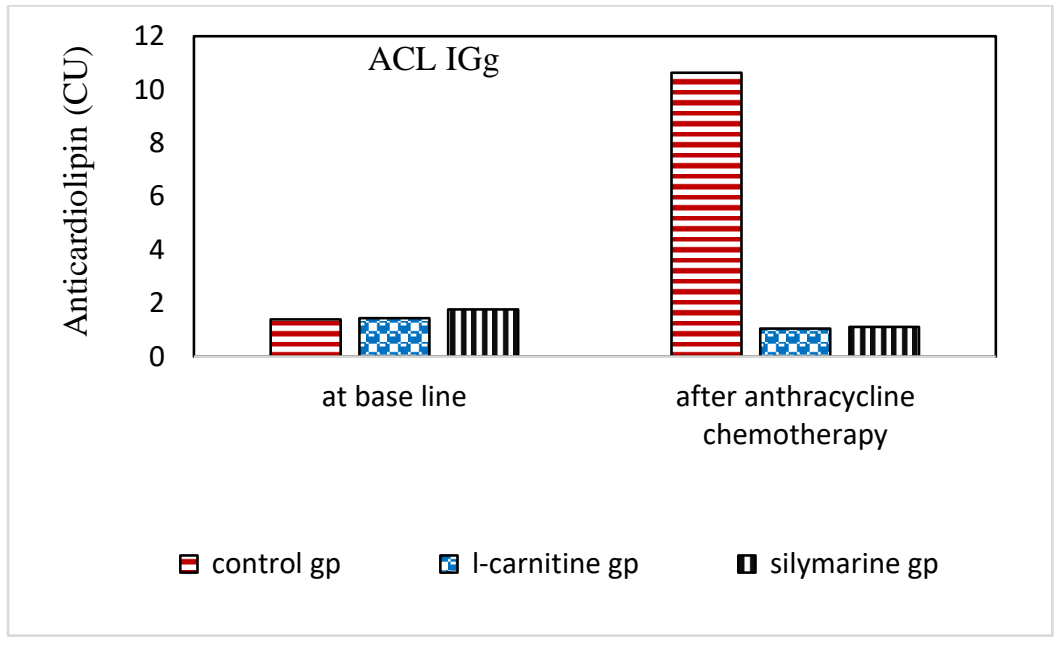

Figure 4: Comparison between studied groups regarding ACL IGg (CU) at base line and after 6 months of chemotherapy 


\section{Discussion}

Anthracycline chemotherapy plays a prominent role in treating many forms of cancer. Cardiotoxic side effects limit their dosing and improved cancer outcomes expose the cancer survivor to increased cardiovascular morbidity and mortality. The basic mechanisms of cardiotoxicity may involve direct pathways for reactive oxygen species generation and topoisomerase 2 as well as other indirect pathways. Cardioprotective treatments are few and those that have been examined include renin-angiotensin system blockade, beta-blockers, or the iron chelator dexrazoxane. Even in the forthcoming era of targeted cancer therapies, the substantial proportion of today's anthracycline-treated cancer patients may become tomorrow's cardiac patient. [2]

According to our knowledge, this current study considered the first study to compare the efficacy and safety of 1 carnitine and silymarin as cardio-protective agents against anthracycline-induced cardiotoxicity in cancer patients.

Our study showed that there is a significant decrease in \% $\mathrm{EF}$ at baseline and after six months in the control group and these results in agreement with Ghaderian et al. 2017 [11]; who found that there was a decline in \% $\mathrm{EF}$ from $68 \pm 7.14$ at baseline to $65.6 \pm 9.3$ after 6 months of doxorubicin administration in Childhood Acute Lymphoblastic Leukemia.

Our study results revealed that there is a significant difference after chemotherapy regarding \% $\mathrm{EF}$ between the control group and L-carnitine group, indicating an increase in ejection fraction in L-carnitine group. As exogenous L-carnitine aids in the resumption of normal oxidative metabolism and restoration of myocardial energy reserves, L-carnitine supplementation has been shown to have favorable effects in CVD patients [14].
Our results are in accordant with Dundar et al [15] who studied the cardioprotective role of L- carnitine in doxorubicin-induced cardiotoxicity and found that EF levels in L- carnitine treated doxorubicin group has been preserved throughout the study. Also, our results show a significant increase in EF when comparing the control group, and silymarin group, it can be due to Silymarin significantly increases the antioxidant capacity of the tissue, and thus, may explain the cardioprotective effect of Silymarin [16]. There were also in vitro and studies in animals showing the antioxidant properties of Silymarin [17]. Silymarin reacts with cell membranes and increases their resistance to harmful influences, most likely through changes in their physicochemical properties, but also interacts with ROS and converts them into less reactive and toxic compounds. In various studies, it was confirmed that Silymarin prevents lipid peroxidation and increases the concentration of glutathione [18]. In another study that evaluating the protective effects of Silymarin on the liver and cardiac side effects of chemotherapy drugs in childhood, acute lymphoblastic leukemia revealed the cardioprotective effect of Silymarin to its ironchelating activity [3], and our results indicate that the iron serum levels in Silymarin group were significantly decreased.

Our results regarding anti-cardiolipin $\mathrm{IgG}$ were that there was a significantly increased in ACL IgG when comparing its levels at baseline and after anthracycline in the control group. Our results are in accordance with Abou Zaid et al. showed a significant increase of ACL IgG in the doxorubicin group compared to control group of white albino rats that indicates the importance of genetic and environmental factors [19]. The ability of aPL to induce a procoagulant phenotype plays a central role in the development of arterial and venous thrombotic manifestations. Inflammation serves as a necessary link between this procoagulant phenotype and actual 
thrombus development [20]. These parameters were used as indicators of cardiotoxicity thrombus initiation in anticardiolipin since doxorubicin tends to accumulate in the nucleus and mitochondria. In heart tissue, mitochondria make up around $50 \%$ of its volume [21]. Doxorubicin has a high affinity to bind the inner mitochondrial membrane and is collected on the matrix side [22]. One of doxorubicin's similarities in the inner mitochondrial membrane is cardiolipin, which has a much higher affinity vs. other lipids in $\begin{array}{llll}\text { mitochondria } & \text { (around } & 80 & \text { times). }\end{array}$ Phosphatidylethanolamine and cardiolipin are adaptors in the hexagonal (HII) phase in existent divalent cations, e.g., doxorubicin, leading to changes in fluidity and functionality of mitochondrial membranes. Doxorubicin inactivates mitochondrial lipid-dependent enzymes, such as NADH dehydrogenase, cytochrome-c oxidase, and cytochrome-c reductase. DOX binds to cardiolipin, causing the inactivation of complex I-III. doxorubicin and NADH/NADH dehydrogenase incubations have been suggested to reduce sequestration at the SR by around $80 \%$. Regarding the 1-carnitine group this result agrees with Paradies et al. 1999 [23] who studied the effect of aging and acetyl-1-carnitine on the pyruvate transport and oxidation in rat heart mitochondria and reported that cardiolipin, a phospholipid located in the inner mitochondrial membrane, is required for the functioning of the isolated pyruvate carrier reconstituted in liposomes. Thus, changes in the mitochondrial cardiolipin content may be responsible for the observed changes in the pyruvate carrier activity with age. LCAR administration to aged rats reversed this loss in cardiolipin concentration to a level not significantly different from young animals. Similar results have been reported by Hagen and colleagues 1998 [24] with hepatocytes isolated from old rats supplemented with ALCAR. No effect was observed on the concentration of other mitochondrial phospholipids.

In the case of the Silymarin group, there is a lack of studies that correlate the effect of Silymarin with serum levels, cardiolipin but this significant decrease in anti-cardiolipin levels after chemotherapy compared to the control group may be interpreted as Silymarin diminished the damage of mitochondrial DNA.

Regarding the iron profile, our results revealed that there is a significant decrease in iron, ferritin, and \% of saturation and a significant increase in total binding capacity when comparing the control group to both Lcarnitine and Silymarin group. There was also, a significant decrease in iron, ferritin, and \% of saturation when comparing the 1-carnitine group to the Silymarin group. Experimental evidence for the role of iron in doxorubicin cardiotoxicity was provided by studies showing that iron overload increases the cardiotoxic effects of the drug. Early studies showed that iron loading exacerbated doxorubicin toxicity in cell culture [25]. Moreover, increasing iron stores in rats by dietary iron loading enhanced doxorubicin cardiotoxicity, and mice lacking HFE, a model that mimics the iron overload found in human hereditary hemochromatosis, showed higher susceptibility to doxorubicin -dependent cardiac damage. It is considered that by forming anthracycline-iron complexes iron potentiates the toxicity of doxorubicin -derived ROS transforming relatively safe species like $\mathrm{O}_{2}{ }^{\circ}$ and $\mathrm{H}_{2} \mathrm{O}_{2}$ into the much more reactive and toxic hydroxyl radical $\mathrm{OH}^{\circ}$ or iron-peroxo complexes that damage DNA, proteins, and lipids [26]. Another possible mechanism linking iron and anthracycline metabolism involves ferritin. While in vitro experiments using purified components suggested that the semiquinone free radical and $\mathrm{O}_{2}{ }^{\circ}$ - produced by redox cycling of doxorubicin quinone moiety 
caused iron release from ferritin [27] the role of ferritin in vivo seems quite opposite. Recent data suggest that the increase in ferritin synthesis induced by DOX could be a defensive mechanism to limit the amount of iron available for ROS production in the heart, and thus prevent oxidative injury, in line with the known antioxidant function of this protein [26]. Moreover, it has been shown that exposure of cardiomyocytes to doxorubicin leads to higher accumulation of iron into ferritin by mechanisms that impair iron release from ferritin and/or lysosomal/proteasomal degradation of this ironstorage protein.

There were some studies designed to investigate the therapeutic activity of silymarin in patients with thalassemia major under conventional iron chelation therapy[28] investigated the therapeutic activity of oral silymarin in patients with thalassemia major under conventional iron chelation therapy in 3 months of randomized, double-blind, clinical trial in 59 betathalassemia major patients who were randomized to receive a silymarin tablet $(140 \mathrm{mg})$ three times per day plus desferrioxamine (group I) or desferrioxamine and placebo (group II). Clinical and laboratory tests were assessed at the beginning and the end of the trial. This study revealed that combined therapy of silymarin and desferrioxamine was well tolerated and more effective than desferrioxamine and placebo in reducing serum ferritin levels. Despite the iron-chelating activity of silymarin suggests its possible application in chelation therapy of iron overload [29]; little is known about the biochemical mechanisms of action of these substances. The biological effects of silymarin are different from other iron chelators, probably due to the antioxidant activity of silymarin, which causes a pro-oxidant effect via iron-catalyzed oxidation with subsequent generation of reactive oxygen species [29]. This result is in agreement with the study that evaluating the protective effects of silymarin on liver and cardiac side effects of chemotherapy drugs in childhood acute lymphoblastic leukemia revealed the cardioprotective effect of silymarin to its iron-chelating activity [11].

\section{Conclusion}

The supplementation of cardioprotective agents such as 1-carnitine, and silymarin provides a new approach to modulate the cardiotoxicity of anthracycline chemotherapy in cancer patients. The use of 1carnitine may be of use in extending the continuous use of anthracycline-containing chemotherapy. The data of the present study show that silymarin is a particularly useful agent as it could enhance myocardial antioxidants when compared to silymarin as a standard commercially available antioxidant, it significantly prevents the heart from anthracyclineinduced oxidative stress, inhibition of lipid peroxidation, iron chelation all of which result in the recuperation of the biological parameters and the integrity of the tissue especially heart tissue. Therefore, it offers useful support to the therapy by acting as a cardioprotective agent and thus prevents the extent of cardiac damage during the treatment of cancer.

\section{Conflicts of interest}

All authors declare that they have no conflicts of interest.

\section{Funding}

This research did not receive any specific grant from funding agencies in the public, commercial, or notfor-profit sectors.

\section{Acknowledgments}

The authors sincerely appreciate the physicians of the oncology Department at Tanta University Hospital, Egypt, for allowing patients to participate in this study and the Faculty of Pharmacy (Girls branch) at Al-Azhar University for supporting this research.

\section{Compliance with Ethics Requirements}


The study was designed and conducted in compliance with the ethical principles of Good Clinical Practice Guidelines and the Declaration of Helsinki [13]. The study protocol was approved by the National Research Ethics Committee [Tanta University Ethical Committee for Clinical Research] approval code (32551/09/2018). Informed written consent was obtained from all individual participants included in the study.

\section{References}

1. Albini A, pennesi G, Donatelli F, Cammarota R, De Flora A, and Noonan DM. Cardiotoxicity of Anticancer Drugs: The Need for Cardio-Oncology and Cardio-Oncological Prevention. J Natl Cancer Inst 2010 Jan 6; 102(1): 14-25

2. Cardinale D, Sandri M. Role of Biomarkers in Chemotherapy-Induced Cardiotoxicity. Sep-Oct 2010;53(2):121129

3. Ghaderian M, Reisi N, Moafi A and Farasat S. Evaluating Protective Effects of Silymarin on Liver and Cardiac Side Effects of Chemotherapy Drugs in Childhood Acute Lymphoblastic Leukemia. Iran J Ped Hematol Oncol. 2017, Vol 7. No 4, 207-215

4. Sure, P.F. Silymarin as a Natural Antioxidant. An Overview of the Current Evidence and Perspectives. Antioxidants 2015, 4, 204-247.

5. Frassová Z, Rudá-Ku erová J. Milk Thistle (Silybum Marianum) as a supportive Phytotherapeutic agent in Oncology. Klin Onkol. 2017, 30(6), 426432.
6. Singal P K, Li T, Kumar D, Danelisen I, and Iliskovic N (2000). Doxorubicininduced heart-failure: mechanism and modulation. Mol. Cell. Biochem., 207: 77-85.

7. EMA. European Medicines Agency. Assessment report. Dexrazoxane-containing medicinal products (EMEA/H/A31/1275) (2011). www.ema.europa.eu/do cs/en_GB/document_library/Referrals_d ocument/Dexrazoxane_31/WC50012034 0.pdf.Google Scholar

8. Sayed -Ahmed MM. Role of carnitine in cancer chemotherapy-induced multiple organ toxicity. Saudi pharmaceutical journal. October 2010;18(4) Pages 195206

9. Flanagan JL, Simmons PA, Vehige J, Willcox MD, Garrett Q: Review Role of carnitine in disease Nutr Metab (Lond). 2010; 7: 30. Published online 2010 Apr 16. doi: 10.1186/1743-7075-7-30

10. Surai, P.F. Silymarin as a Natural Antioxidant. An Overview of the Current Evidence and Perspectives. Antioxidants 2015, 4, 204-247.

11. Frassová Z, Rudá-Ku erová J. Milk Thistle (Silybum Marianum) as a supportive Phytotherapeutic agent in Oncology. Klin Onkol. 2017, 30(6), 426432.

12. Zou, H.; Zhu, X.X.; Zhang, G.B.; Ma, Y.; Wu, Y.; Huang, D.S. Silibinin: an old drug for hematological disorders. Oncotarget 2017 Jul 11; 8(51): 89307-89314. 
13. World Medical Association Inc. Declaration of Helsinki. Ethical principles for medical research involving human subjects. J Indian Med Assoc 2009; 107:403-405.

14. Flanagan JL, Simmons PA, Vehige J, Willcox MD, Garrett Q: Review Role of carnitine in disease Nutr Metab (Lond). 2010; 7: 30. Published online 2010 Apr 16. doi: 10.1186/1743-7075-7-30

15. Dundar HA, Kiray M, Kir M, Kolatan E, Bagriyanik A,et al. Protective Effect of Acetyl-L-Carnitine Against Doxorubicininduced Cardiotoxicity in Wistar Albino Rats. Archives of Medical Research 47 (2016) 506e514

16. Šimánek, V.; Škottová, N.; Bartek, J.; Psotova, J.; Kosina, P.; Balejova, L.; Ulirhova, J. Extract from Silybum marianum as a nutraceutical: A doubleblind placebo-controlled study in healthy young men. Czech J. Food Sci. 2001, 19, 106-110.

17. Polyak, S.J.; Morishima, C.; Lohmann, V.; Pal, S.; Lee, D.Y.W.; Liu, Y.; Graf, T.N.; Oberlies, N.H. Identification of hepatoprotective flavonolignans from silymarin. Proc. Natl. Acad. Sci. USA 2010, 107, 5995-5999.

18. Injac, R.; Perse, M.; Obermajer, N.; Djordjevic-Milic, V.; Prijatelj, M.; Djordjevic, A.; Cerar, A.; Strukelj, B. Potential hepatoprotective effects of fullerenol $\mathrm{C} 60(\mathrm{OH}) 24$ in doxorubicininduced hepatotoxicity in rats with mammary carcinomas. Biomaterials 2008, 29, 3451-3460.
19. Abou Zaid OAR, Ibraheem A, Osama HA et al. Biochemical Study on Attenuating the Cardiotoxic Effect of Doxorubicin through Transforming into Mesoporous Nanoparticles Form. bvmj, 35(1): 342-351, Sept., 2018

20. Rohan, W.; and Silvia, S. P.; 2011: Pathophysiology of the antiphospholipid antibody syndrome. Auto Immun Highlights. ; 2(2): 35-52.

21. Jung K, Reszka R. Mitochondria as subcellular targets for clinically useful anthracyclines. Advanced Drug Delivery Reviews. Jul 2001;49(1-2):87-105

22. Sorensen JC, Cheregi BD, Timpani CA, Nurgali K, Hayes A, Rybalka E. Mitochondria: Inadvertent targets in chemotherapy-induced skeletal muscle toxicity and wasting? Cancer Chemotherapy and Pharmacology. Oct 2016;78(4):673-683

23. Paradies G, Petrosillo G, Gadaleta MN et al. The effect of aging and acetyl-Lcarnitine on the pyruvate transport and oxidation in rat heart mitochondria. FEBS Letters 454 (1999) 207209

24. Hagen, T.M., Ingersoll, R.T., Wehr, C.M., Lykkesfeldt, J., Vinarsky, V., Bartholomew, J.C., Song, M. and Ames, B.N. (1998) Proc. Natl. Acad. Sci. USA 95, 95629566

25. Link G, Tirosh R, Pinson A, Hershko C Role of iron in the potentiation of anthracycline cardiotoxicity: identification of heart cell mitochondria as a major site of iron-anthracycline $\mathrm{J}$ Lab Clin Med. 1996 Mar; 127(3):272-8. 
26. Arosio P, Levi S. Cytosolic and mitochondrial ferritins in the regulation of cellular iron homeostasis and oxidative damage. Biochim Biophys Acta. 2010 Aug; 1800(8):783-92.

27. Thomas CE, Aust SD. Reductive release of iron from ferritin by cation free radicals of paraquat and other bipyridyls. J Biol Chem. 1986 Oct 5; 261(28):13064-70.

28. Gharagozloo M, Moayedi B, Zakerinia M, Hamidi M, Karimi M, et al. (2009)
Combined therapy of silymarin and desferrioxamine in patients with betathalassemia major: a randomized double-blind clinical trial. Fundam Clin Pharmacol 23: 359-365.

29. Gharagozloo M, Khoshdel Z, Amirghofran Z (2008) The effect of an iron (III) chelator, silybin, on the proliferation and cell cycle of Jurkat cells: a comparison with desferrioxamine. Eur J Pharmacol 589: 1-7. 